\title{
L'Europe et le monde germanique (époque moderne et contemporaine)
}

\author{
Jacques Le Rider
}

\section{OpenEdition}

\section{Journals}

Édition électronique

URL : https://journals.openedition.org/ashp/1197

DOI : 10.4000/ashp.1197

ISSN : 1969-6310

\section{Éditeur}

Publications de l'École Pratique des Hautes Études

\section{Édition imprimée}

Date de publication : 1 octobre 2011

Pagination : 229-230

ISSN : 0766-0677

\section{Référence électronique}

Jacques Le Rider, «L'Europe et le monde germanique (époque moderne et contemporaine) », Annuaire de l'École pratique des hautes études (EPHE), Section des sciences historiques et philologiques [En ligne],

142 | 2011, mis en ligne le 27 juillet 2011, consulté le 06 juillet 2021. URL : http:// journals.openedition.org/ashp/1197; DOI : https://doi.org/10.4000/ashp.1197 


\title{
L'EUROPE ET LE MONDE GERMANIQUE (ÉPOQUE MODERNE ET CONTEMPORAINE)
}

\author{
Directeur d'études : M. Jacques Le RIDER
}

Programme de l'année 2009-2010 : L'école viennoise d'histoire de l'art (1854-1938).

\section{L'École viennoise d'histoire de l'art (1854-1938)}

L'histoire de l'École viennoise d'histoire de l'art commence le 9 novembre 1852, avec la nomination de Rudolf Eitelberger au poste de professeur d'histoire et archéologie de l'art à l'université de Vienne. Cette création d'une chaire d'histoire de l'art s'inscrit dans le cadre de la politique de réforme menée par le comte Leo Thun-Hohenstein, nommé ministre de l'Instruction publique en 1849 (l'Institut für Österreichische Geschichtsforschung est fondé en 1854). En 1864, Eitelberger est le fondateur et le premier directeur du Österreichisches Museum für Kunst und Industrie. La création de ce nouveau musée, qui sera complété en 1868 par une Kunstgewerbeschule, répond à projet de formation de la culture et du goût artistique contemporains. La collection d'art ancien est destinée à inspirer la production du temps présent. Le musée entend réunir les artistes, les industriels et maîtres artisans, et le grand public. Eitelberger est particulièrement attentif aux modèles anglais (mouvement «Arts \& Crafts », musée de South Kensington créé à la suite de l'exposition universelle de Londres en 1851). On peut rapprocher les conceptions d'Eitelberger de celles de Gottfried Semper : pour le premier, c'est le programme d'éducation artistique destiné à compléter la Bildung qui est primordial..

Moriz Thausing, étudiant à l'Institut für österreichische Geschichtsforschung à partir de 1858, élève d'Eitelberger, qui entre à l'Albertina en 1864 (il en prend la direction en 1864), professeur extraordinarius à l'université de Vienne en 1873, ordinarius en 1879, défend pour sa part une conception plus exclusivement scientifique de l'histoire de l'art. Sa leçon inaugurale d' octobre 1873 s'intitule Die Stellung der Kunstgeschichte als Wissenschaft. Contrairement à son maître Eitelberger, Thausing n'entretient pas de relations étroites avec l'art et l'industrie d'art contemporains. Il apprécie particulièrement les travaux et la méthode de Giovanni Morelli. En 1883, il refuse l'invitation de la ville de Vienne à participer au jury chargé de choisir les fresques destinées à décorer la salle d'assemblée du conseil municipal (Gemeinderatssaal), estimant que trop d'artistes font partie du jury et pas assez de savants. Il fustige « L'Entrée de Charles Quint à Anvers » et « Venise rend hommage à Caterina Cornaro », du peintre à la mode Hans Makart, pour leurs inexactitudes historiques.

Après une séance consacrée à Albert Ilg (1847-1896), élève d'Eitelberger, nommé en 1884 directeur de la collection d'armes et d'objets d'art du Kunsthistorisches Hofmuseum, auteur d'une série de pamphlets critiquant la décadence contemporaine, partisan d'une conception stricte selon laquelle les antiquités sont affaire d'État et ne sauraient être traitées comme des objets du commerce de l'art, on a étudié de 
manière plus approfondie le continuateur de Thausing, Franz Wickhoff. Devenu en 1880 conservateur au Österreichisches für Kunst und Industrie fondé par Eitelberger, professeur extraordinarius à l'université de Vienne en 1885, puis ordinarius, successeur de Thausing, en 1891, Wickhoff est une des grandes figures de l'histoire de l'art qui ont illustré l'École viennoise. Wickhoff est connaisseur de l'art contemporain, il fait la théorie de l'impressionnisme et du japonisme. Il intervient dans la controverse suscitée par la première des trois fresques commandées à Klimt pour l'université de Vienne, prenant la défense de l'artiste dans une conférence du 9 mai 1900, devant la Société de philosophie de l'université de Vienne, sur le thème : « Was ist häßlich? ». Ces analyses de l'art moderne vont de pair avec la réévaluation de l'art du Bas-Empire romain. La fameuse étude de 1895 consacrée à la Wiener Genesis, éditée en collaboration avec le philologue Wilhelm von Hartel, renouvelle la théorie des relations entre le texte et l'image en même temps qu'il analyse les éléments de rupture avec l'esthétique classique. La réfutation de la notion de décadence influencera directement Riegl.

Alois Riegl a fait des études de droit, puis d'histoire. En 1881-1883, il a été étudiant à l'Institut für Österreichische Geschichtsforschung. En 1885, quand Wickhoff devient ao. Prof., Riegl prend sa succession à la tête de la Textilsammlung du Österreichisches Museum für Kunst und Industrie. En 1894, il est nommé professeur extraordinarius, puis en 1897 ordinarius à l'université de Vienne. En 1902, il est nommé conservateur général et président de la Commission centrale des monuments artistiques et historiques d'Autriche-Hongrie. Son ouvrage Stilfragen. Grundlegungen zu einer Geschichte der Ornamentik, 1893, place les arts décoratifs et l'histoire de l'ornementation au premier plan de l'histoire de l'art et introduit une nouvelle définition de la notion de style, inscrite dans le prolongement d'une anthropologie historique de la perception (Wahrnehmung). Son étude Spätrömische Kunstindustrie nach den Funden in Österreich-Ungarn, im Zusammenhang mit der Gesamtentwicklung der bildenden Kunst bei den Mittelmeervölkern, de 1901, témoigne de l'importance de l'archéologie autrichienne et, prenant la suite de l'étude de Wickhoff sur la Wiener Genesis, réévalue les périodes dites de transition, de décadence ou de maniérisme que l'esthétique normative avait tendance à négliger.

Plusieurs séances ont été consacrées à d'autres aspects de l'œuvre de Riegl : Le portrait de groupe hollandais, de 1902, La culte moderne des monuments, de 1903, La formation de l'art baroque à Rome (ouvrage posthume publié en 1908).

Les dernières conférences de l'année 2009-2010 ont été consacrées à l'œuvre de l'historien d'art Max Dvorák, un sujet que M. Hans Aurenhammer, professeur à l'université de Francfort, directeur d'études invité, a approfondi dans ses deux premières conférences, avant de retracer, dans les deux suivantes, l'histoire de l'École viennoise d'histoire de l'art dans les années 1920 et 1930. 